\title{
COMPARISON OF CHROMOSOMAL REARRANGEMENTS IN BONE MARROW CELLS AND BLAST TRANSFORMED B-CELLS IN RELAPSE OF B-CELL CHRONIC LYMPHOCYTIC LEUKEMIA/ SMALL LYMPHOCYTIC LYMPHOMA
}

\author{
S.V. Andreieva ${ }^{1,}$ *, K.V. Korets ${ }^{2}$, O.E. Ruzhinska ${ }^{2}$, I.M. Skorokhod ${ }^{2}$, O.G. Alkhimova ${ }^{3}$ \\ ${ }^{1}$ State Institution "Institute of Haematology and Transfusiology of NAMS of Ukraine", \\ Kyiv 04060, Ukraine \\ ${ }^{2}$ Government Institution "The Scientific-Practical Children's Cardiac Centre of the Ministry of Health \\ of Ukraine” (UCCC), Kyiv 04050, Ukraine \\ ${ }^{3}$ Institute of Molecular Biology \& Genetics of NAS of Ukraine, Kyiv 03680, Ukraine
}

\begin{abstract}
Aim: The genetic mechanisms of resistance to chemotherapy in B-cell chronic lymphocytic leukemia/small lymphocytic lymphoma (B-CLL/SLL) are not clear. We aimed to determine the peculiarities of abnormal karyotype formation in bone marrow (BM) cells and peripheral blood (PB) blast transformed B-cells in relapse of B-CLL/SLL. Materials and Methods: Cytogenetic GTG banding technique and molecular cytogenetic in interphase cells (i-FISH) studies of BM cells and PB blast transformed B-lymphocytes were performed in 14 patients (10 males and 4 females) with B-CLL/SLL. Results: The results of karyotyping BM and PB cells revealed the heterogeneity of cytogenetic abnormalities in combined single nosological group of B-CLL/SLL. In PB B-cells, chromosome abnormalities related to a poor prognosis group were registered 2.5 times more often than in BM cells. Additional near tetraploid clones that occurred in $\mathbf{5 7 . 1 \%}$ cases were the peculiar feature of BM cell karyotypes. Chromosomal rearrangements characteristic of the group of adverse cytogenetic prognosis were revealed in all cases from which in 2 cases by karyotyping BM cells, in 6 cases in PB B-cells and in 8 cases by the i-FISH method in BM cells, i.e. their detection frequency was 3 times higher in PB B-cells and 4 times higher when analyzing by i-FISH in BM cells. Conclusions: Mismatch in abnormal karyotypes in BM and PB B-cells by the presence of quantitative and structural chromosomal rearrangements may be indicative of simultaneous and independent processes of abnormal clone formation in the lymph nodes and BM hematopoietic cells. Accumulation the information about previously unidentified chromosomal rearrangements in relapse of the disease may help to understand the ways of resistance formation to chemotherapy. Key Words: B-cell chronic lymphocytic leukemia, lymphoma of small lymphocytes, relapse, bone marrow, blast transformed B-cells, chromosomal abnormalities.
\end{abstract}

B-cell chronic lymphocytic leukemia (B-CLL) is the most common type of leukemia among the population of Europe and North America countries, and makes up approximately $30.0 \%$ of all neoplasia of hematopoietic and lymphoid tissues. In Ukraine today, about 11,000 people are diagnosed and registered with CLL. The WHO classification of tumors of hematopoietic and lymphoid tissues (2016) combines B-CLL with small lymphocytic lymphoma (SLL) considered as a single nosology form [1]. Cytomorphological identity characteristics, immunophenotype and the results of molecular genetic analysis serves as a basis for such unified B-CLL/SLL entity. Both diseases have a clonal origin from B-cells while in SLL, substrate cells are not detected in the peripheral blood (PB) [2]. In B-CLL, primary genetic rearrangements may occur in B-cells of bone marrow (BM). Further repeated antigenic stimulation leads to the accumulation of additional genetic rearrangements resulting in leukemia development. Evaluation of genetic abnormalities, in particular

Submitted: April 27, 2017.

Correspondence: $\quad$ Fax: +38 (044) 4402722

E-mail: office@immd.kiev.ua

Abbreviations used: B-CLL - B-cell chronic lymphocytic leukemia; $\mathrm{BM}$ - bone marrow; FISH - fluorescence in situ hybridization; i-FISH - interphase nuclei fluorescence in situ hybridization; PB peripheral blood; SLL - small lymphocytic lymphoma. cytogenetic, conducted at diagnosis, is necessary for better understanding of the biological characteristics of the tumor and the prognosis, being advantageous for the stratification of patients in clinical risk groups [3].

Recently, for evaluation of genetic rearrangements, the fluorescence in situ hybridization (FISH) of interphase nuclei FISH (i-FISH) has been commonly used, i.e. analysis of chromosomal rearrangements is carried out in non-dividing cells. However, this method has limitations since only those genome regions are identified for which FISH probes are available.

Comparison of chromosomal abnormalities with the disease peculiarities and the response to chemotherapy allowed distinguishing between three groups of cytogenetic prognosis. To the group with favorable prognosis, the patients with cytogenetically normal karyotype and del(13q) detectable by i-FISH are included. The group with adverse prognosis includes the patients who have been registered for following changes: del(11q) with the loss of ATM gene and del(17p) with the loss of a tumor suppressor gene TP53. Malignant cells, which carry these abnormalities, are characterized by resistance to conventional chemotherapy with purine analogs and alkylating agents. In addition, the patients with del(6) (q21-23) are included in this group. Trisomy 12 as well as the abnormalities not listed in the previous two groups constitute a group of intermediate prognosis [4-6]. 
The complexity of standard cytogenetic study of metaphase chromosomes in BM cells by G-banding is associated with low proliferative activity of substrate cells and their accumulation in $G_{0} / G_{1}$ phase of cell cycle. For obtaining a population of dividing cells, the stimulation by B-cell mitogens such as pokeweed mitogen (PWM), 12-O-tetradecanoyl-phorbol-13-acetate (TPA), lipopolysaccharides are traditionally used. However, the frequency of abnormal clones in such blast transformed B-lymphocytes makes up only 40 $50 \%$ [7-9]. To improve the effectiveness of cytogenetic studies, the attempts were made to use other stimulators of the cell cycle. Among the new mitogens, the CpGoligodeoxynucleotide DSP30 (CpG-ODN) [10] is used. Thus, detection of chromosomal abnormalities increases to $80 \%$ of cases. Moreover, the rearrangements have been registered that had not been described previously.

Therefore, we aimed to determine the patterns of abnormal karyotypes in BM cells and PB blast transformed B-lymphocytes in relapse of B-CLL/SLL.

\section{MATERIALS AND METHODS}

Patients. Cytogenetic and molecular cytogenetic studies of BM cells and blast transformed B-lymphocytes were performed in 14 patients ( 10 males and 4 females) with B-CLL/SLL. The average age in the group was 53.3 years (range 40 to 65 years). The leukocyte count in patients examined was recorded within $7.4-78.4 \cdot 10^{9} /$, hemoglobin - 121.0-155.0 g/l, thrombocytes 106.0-294.0 $10^{9} /$, the lymphocyte count in PB ranged from 69.0 to $98.0 \%$. The patients gave written informed consent for the publication of their data analyses.

Cytogenetic studies. Metaphase chromosome of $\mathrm{BM}$ cells were prepared according to the conventional method [11] after 24 h culture in RPMl 1640 medium supplemented with $20 \%$ fetal bovine serum followed by G-bands staining (GTG technique). Metaphase chromosomes of PB blast transformed B-lymphocytes were prepared after $72 \mathrm{~h}$ of culture in RPMl 1640 medium containing $10 \%$ fetal bovine serum, $3.6 \mathrm{mg} / \mathrm{ml}$ gentamicin and CpG-ODN mitogen at a final concentration of $2.5 \mu \mathrm{g} / \mathrm{ml}$ [12].

Identified chromosomal abnormalities were described according to the International nomenclature of human chromosomes ISCN 2013 [13]. Only clonal chromosomal abnormalities were taken into account in the study. The karyotype was considered as normal when no less than 20 analyzed and 10 karyotyped metaphase plates showed no chromosomal abnormalities.

Table. Comparison of results of cytogenetic and molecular cytogenetic studies of BM cells and PB blast transformed B-lymphocytes in relapse of B-CLL

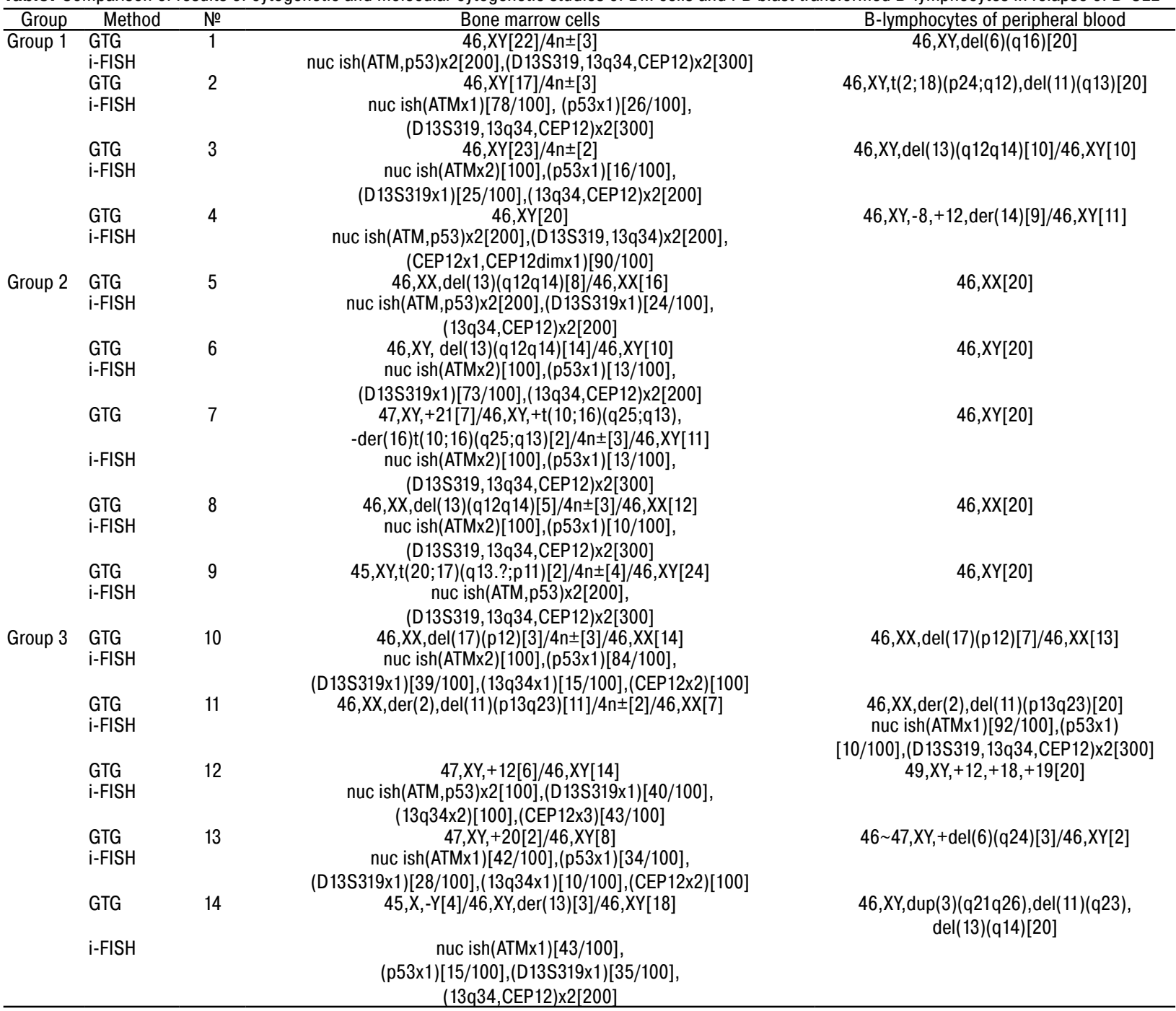


i-FISH was performed after 24-h or 72-h culture. For this purpose, two sets were used: Vysis LSI p53 (locus specific probe for TP53 gene detection located in the disk 17p13.1), LSI ATM (probe for registration ATM gene located in the disk 11q22.3) and LSI D13S319 (disc 13q14), LSI 13q34, CEP12 (centromeric region of chromosome 12) (Abbott, USA). The error of method was $8.0 \%$.

\section{RESULTS ANS DISCUSSION}

The results of BM cells and PB blast transformed $B$-lymphocytes karyotyping were divided into three groups according to the clone structure. For all cases, in parallel, the molecular cytogenetic studies were carried out in i-FISH (Table). A distinctive feature of a half of BM cell karyotypes was the presence of additional near tetraploid clone (50\%) (No. 1-3, 8-11). Formation of abnormal clones both in BM (Fig. 1) and PB (Fig. 2) was due to quantitative (monosomy, trisomy, marker chromosomes) and structural chromosomal rearrangements (deletions, translocations, derivative chromosomes).

Four cases were related to the first group, where normal (N) (No. 4) and the mosaic karyotypes (normal and near teraploid clones $-\mathrm{N} / 4 \mathrm{n} \pm$ ) (No. 1-3) were registered in BM cells. In addition, microdeletions of ATM genes, TP53 (No. 2) and 13q14, TP53 (No. 3) were identified in this group by i-FISH method that was confirmed by karyotyping PB cells of these patients, namely: del(11)(q13) (No. 2) and del(13)(q12q14) (No. 3). At the same time, in PB B-cells, the karyotypes were represented by the pseudodiploid clones formed as a result of deletions del(6)(q16) (№ 1), del(11)(q13) (No. 2), del(13)(q12q14) (No. 3), the presence of the derivative chromosome 14 (No. 4), and unbalanced quantitative abnormalities monosomy 8 and trisomy 12 (No. 4). In 2 cases (No. 3, 4) in $\mathrm{PB}$, the mosaic karyotype was registered, wherein the second clone was represented by cytogenetically normal karyotype, possibly due to the presence of normal lymphocytes. Interesting results were obtained in cases No. 1 and 4. On the one hand, the study of BM showed the cytogenetically normal karyotype and absence of deletion in the regions carrying the tumor suppressor genes. On the other hand, the presence of chromosomal abnormalities in PB in these patients may indicate the origin of these abnormal clones outside the BM and is likely to evidence in favor of SLL with the leukemization.

In the second group, the reverse situation was observed, namely, a normal karyotypewas revealed in B-cells of PB while in BM cells almost all abnormal clones were pseudodiploid. Thus, in 3 cases the abnormal clone was formed by an unbalanced structural rearrangement, the interstitial deletion del( 13)(q12q14) (No. 5, 6, 8); in 2 other cases, trisomy 21 and unbalanced translocation, $\mathrm{t}(10 ; 16)$ (q25;q13) (No. 7) and t(20;17)(q13;p11.?) (No. 9) were detected. The absence of abnormal karyotypes in B-cells of $\mathrm{PB}$ and their presence in $\mathrm{BM}$ cells may indicate that the $\mathrm{BM}$ is the source of the abnormal clone formation.

In the third group, the clonal abnormalities were revealed both in BM cells and B-cells of PB. Thus, in the first two cases, the abnormal clones in $\mathrm{BM}$ as well as in PB consisted of identical structural chromosomal

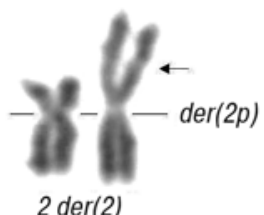

$13 \operatorname{der}(13)$

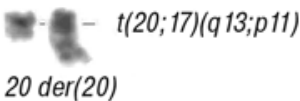

Fig. 1. Structural chromosomal rearrangements of BM cells in relapse of B-CLL/SLL
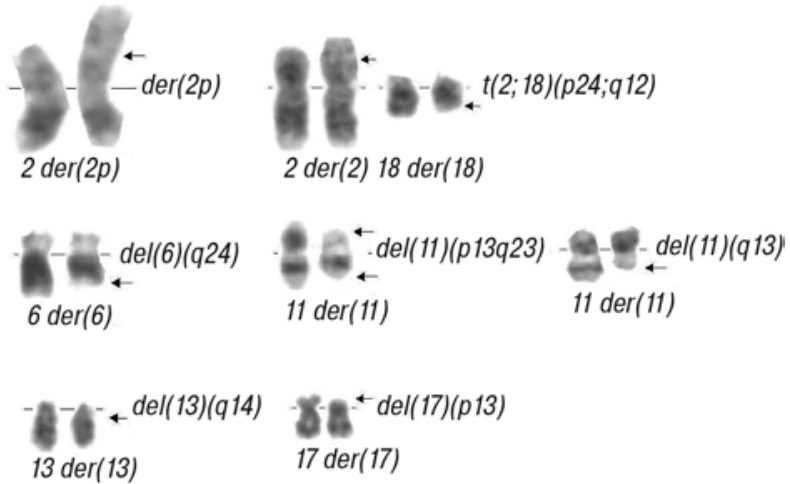

Fig. 2. Structural chromosomal rearrangements of $P B$ blast transformed cells in relapse of B-CLL/SLL

rearrangements: del(17)(p12) (No. 10) and der(2), del(11) (p13q23) (No. 11). In all cases, the mosaic karyotype with presence of cytogenetically normal clone was observed in BM cells, while in PB the presence of cytogenetically normal clone was detected only in 2 cases out of 5 (No. 10, 13), which may indicate the relative safety of the cellular pool of BM hematopoietic cells in the patients. In the case No. 12, the BM cells and PB B-cells shared the trisomy of chromosome 12, which is typical for B-CLL, and is registered with the karyotyping in $10-15 \%$ cases, and in 30\% - by FISH [14]. However, in cases No. 12-14, the numerical and structural clonal abnormalities in BM and PB did not coincide. Such diversity appears to be due to the existence of additional mechanisms for the formation of the populations of malignant cells in B-CLL/SLLalso taking into account the role of microenvironmental factors.

In general, the chromosomal abnormalities in BM cells in a group with an adverse cytogenetic prognosis were detected in 2 cases by karyotyping (No. 10, 11) and in 9 cases using i-FISH (No. 2, 3, 6, 7, 8, 10, 11, 13 and 14), two of which were identified in the cytogenetically normal karyotypes. Abnormalities related to the group of a favorable prognosis were detected in 7 cases (No. 1-6, 8) on karyotyping results, wherein results of FISH for 4 patients (No. 2, 3, 6, 8) ranked to a group of poor prognosis mainly due to the presence of del(17). 8 patients (No. 5-9, 12-14) after karyotyping were assigned to the group of intermediate prognosis, 5 of them 
being in the group of poor prognosis according to the results of FISH analysis (No. 6, 7, 8, 13, 14).

In PB B-cells, abnormalities ranked to a poor prognosis group by karyotyping results were reported in 6 cases (No. 1, 2, 10, 11, 13, 14). The group attributed to the favorable cytogenetical prognosis included 6 cases (No. 3, 5-9). The chromosomal aberrations belonging to the group of intermediate prognosis (No. 4, 12) were revealed in 2 cases.

Thus, the results of karyotyping BM and PB cells revealed the heterogeneity of cytogenetic abnormalities in a single nosological group B-CLL/SLL. Based on karyotyping, in PB B-cells chromosome abnormalities related to a poor prognosis group were registered 2.5 times more often than in BM cells. However, results of FISH analysis in BM cells allowed us to increase the number of patients with a poor prognosis that occurs mainly due to the presence of del(11) and del(17), i.e. associated with a loss of tumor suppressor genes [15-20].

Therefore, the results of karyotyping have shown heterogeneity of cytogenetic abnormalities by karyotype structure, ploidy, numerical and structural chromosome rearrangements in a single nosological group of B-CLL/SLL. Additional near-tetraploid clones that occurred in $57.1 \%$ cases were the peculiar feature of the BM cell karyotypes. Chromosomal rearrangements characteristic of the group of adverse cytogenetic prognosis were revealed in all cases from which 2 cases - by karyotyping of BM cells, 6 - in PB B-cells and $8-$ by the i-FISH method in BM cells, i.e. their detection frequency was 3 times higher in PB B-cells and 4 times higher when analyzing by i-FISH in BM cells. These findings suggest that all three methods should be used for full information concerning the genetic rearrangements both in BM cells and B-cells in B-CLL/SLL. Ambiguous are the findings of the normal karyotype in B-cells of $\mathrm{PB}$ while karyotype in BM cells was abnormal. Accumulation of the information about previously unidentified chromosomal rearrangements in relapse of the disease may help to understand the ways of resistance formation to chemotherapy. Mismatch in abnormal karyotypes in BM and PB B-cells by the presence of quantitative and structural chromosomal rearrangements may be indicative of simultaneous and independent processes of abnormal clone formation in the lymph nodes and BM hematopoietic cells.

\section{ACKNOWLEDGEMENTS}

We thank Prof. Danylo Gluzman, R.E. Kavetsky Institute of Experimental Pathology, Oncology and Radiobiology of the NAS of Ukraine, for the confirmation of diagnosis and stage of the disease.

\section{REFERENCES}

1. Swerdlow SH, Campo E, Stefano A, et al. Updated WHO classification of hematological malignancies. The 2016 revision of the World Health Organization classification of lymphoid neoplasms. Blood 2016; 127: 2375-90.

2. Gluzman DF. Chronic lymphocytic leukaemia: Diagnostic oncohematology. Kyiv: DIA, 2011; 7: 125-38 (in Russian).

3. Hastings R, Howell R, Betts D, et al. Guidelines and quality assurance for acquired cytogenetic. A common European framework for quality assessment for banded chromosome studies and molecular cytogenetic investigations of acquired abnormalities. E.C.A. Permanent Working Group for Cytogenetics and Society. Eur Cytogenet Association Newsletter 2013; 31: 7-21.

4. Motyckova M, Zak P, Vroblova V, et al. Prognostic markers in chronic lymphocytic leukemia. Vnitr Lek 2011; 57: 847-57 (in Czech).

5. Riches JC, O'Donovan CJ, Kington SJ, et al. Trisomy 12 chronic lymphocytic leukemia cells exhibit upregulation of integrin signals that is modulated by NOTCH1 mutations. Blood 2014; 123: 4101-10.

6. Eichhorst B, Robak T, Montserrat E, et al. Chronic lymphocytic leukemia: ESMO Clinical Practice Guidelines for diagnosis, treatment and follow-up. Ann Oncol 2015; 26: 78-84.

7. Gahrton G, Robert KH, Friberg K, et al. Nonrandom chromosomal aberrations in chronic lymphocytic leukemia revealed by polyclonal B-cell-mitogen stimulation. Blood 1980; 56: 640-7.

8. Juliusson G, Oscier DG, Fitchett M, et al. Prognostic subgroups in B-cell chronic lymphocytic leukemia defined by specific chromosomal abnormalities. N Engl J Med 1990; 323: 720-4.

9. Geiser CH, Philip P, Christensen BE, et al. In B-cell chronic lymphocytic leukemia chromosome 17 abnormalities and not trisomy 12 are the single most important cytogenetic abnormalities for the prognosis: a cytogenetic and immunophenotypic study of 480 unselected newly diagnosed patients. Leuk Res 1997; 21: 1011-23.

10. Stěpanovská K, Vaňková G, Némethová V, et al. Chromosome banding analysis of peripheral blood lymphocytes stimulated with IL2 and CpG oligonucleotide DSP30 in patients with chronic lymphocytic leukemia. Klin Onkol 2013; 26: 263-70 (in Czech).

11. Rooney DE Czepulkovsky BH. Human cytogenetics. Malignancy and Acquired Abnormalities: a practical approach. Oxford New York Tokyo: Oxford University Press, 1995. 25 p.

12. Andreieva SV, Korets KV. Method for prognosis of B-cell chronic lymphocytic leukemia. Patent N97826 UA, MPK A61V 5/00, G01N 33/48. Nu2014 10373, 2015, Bull 7.

13. International System for Human Cytogenetic Nomenclature. In: Shaffer LG, McGowan-Jordan J, Schmid M, eds. Recommendations of the International Standing Committee on Human Cytogenetic Nomenclature. Basel: S Karger, 2013. 140 p.

14. Thompson PA, Ferrajoli A, O'Brien S, et al. Trisomy 12 is associated with an abbreviated redistribution lymphocytosis during treatment with the BTK inhibitor ibrutinib in patients with chronic lymphocytic leukaemia. British J Haemat 2015; 170: 125-8.

15. Stilgenbauer $S$, Bullinger $L$, Benner $A$, et al. Incidence and clinical significance of $6 \mathrm{q}$ deletions in B cell chronic lymphocytic leukemia. Leukemia 1999; 13: 1331-4.

16. Calin GA, Dumitru CD, Shimizu M, et al. Frequently deletions and down-regulation of micro-RNA genes miR15 and miR16 at 13q14 in chronic lymphocytic leukemia. Proc Nat Acad Sci USA 2002; 99: 15524-9.

17. Mraz M, Pospisilova S, Malinova K, et al. MicroRNAs in chronic lymphocytic leukemia pathogenesis and disease subtypes. Leuk Lymphoma 2009; 50: 506-9.

18. Zenz T, Habe S, Denzel T, et al. Detaled analysis of p53 pathway defects in fludarabine-refractory chronic lymphocytic leukemia (CLL): dissecting the contribution of $17 p$ deletion, TP53 mutation, p53-p21 dysfunction, and miR34a in a prospective clinical trial. Blood 2009; 114: 2589-97.

19. Klein U, Lia M, Crespo M, et al. The DLEU2/mi$\mathrm{R} 15 \mathrm{a} / 16-1$ cluster controls B cell proliferation and its deletion leads to chronic lymphocytic leukemia. Cancer Cell 2010; 17: 28-40.

20. Fabbri M, Bottoni A, Shimizu M, et al. Association of microRNA/TP53 feedback circuitry with pathogenesis and outcome of B-cell chronic lymphocytic leukemia. JAMA 2011; 305: 59-67. 